\title{
Downregulation of nuclear protein-1 induces cell cycle arrest in G0/G1 phase in glioma cells in vivo and in vitro via P27
}

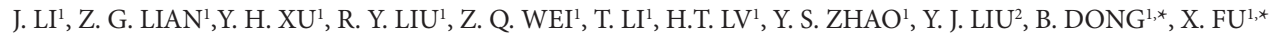 \\ ${ }^{1}$ Department of Neurosurgery, First Affiliated Hospital of Dalian Medical University, Dalian Medical University, Dalian, Liaoning, China; \\ ${ }^{2}$ Department of Interventional Therapy, First Affiliated Hospital of Dalian Medical University, Dalian Medical University, Dalian, Liaoning, China
}

*Correspondence: stocktondb@163.com; fuxin1127@126.com

Received August 14, 2019 / Accepted November 6, 2019

\begin{abstract}
Nuclear protein-1 (NUPR1), also named as p8 or Com1, has been since found overexpressed in several human malignant tumor cells, such as glioma. NUPR1 also regulates cell cycle progression, however, the role of NUPR1 in regulating glioma cell cycle remains poorly understood. Knockdown efficiency of U87 and U251 cells infected with the lentiviral vector was detected by quantitative real-time PCR and western blot in vitro and in vivo. Flow cytometry and western blot were used to explore a mechanism by which NUPR1 modulates cell cycle in U87 and U251 cells. Immunohistochemistry was applied to detect expression levels of P27, CDK2, and cyclin E in human glioma tissues with NUPR1 positive expression and tumorigenesis in nude mice. We confirmed that the downregulation of NUPR1 arrested the cell cycle in the G0/G1 phase in U87 and U251 cells in vitro. Furthermore, the expression level of P27 was increased, and CDK2 and cyclin E were decreased upon silencing NUPR1 expression in vitro and in vivo. In conclusion, the knockdown of NUPR1 induces cell cycle arrest in the G0/G1 phase in glioma cells via P27.
\end{abstract}

Key words: glioma, NUPR1, P27, cell cycle

Glioma, the most common and malignant primary brain tumor, is characterized by a low cure rate and a high relapse rate. Even considering individual treatments, the survival time is still poor [1]. To date, more and more biomarkers were found to be related to the occurrence, growth, invasion, and prognosis of glioma but not be closely and highly specific, such as isocitrate dehydrogenase (IDH), O6-methylguanineDNA methyltransferase (MGMT), and 1P19q (co-deletion) loss [2].

NUPR1 (p8 or Com1), detected in pancreatic acinar cells of rats caused by acute pancreatitis, is located on human chromosome $16 \mathrm{p} 11.2$ and its gene encodes a protein with a theoretical molecular mass of 8872.7 Da [3]. NUPR1 induces some biochemical properties similar to HMG proteins because of its structural similarity to HMG proteins [4]. There is no significant homology between NUPR1 and other proteins of known function, such as isoelectric point, hydrophilicity plot, resistance to denaturation after heating at $100^{\circ} \mathrm{C}$ and the charge distribution along the polypeptide [5]. NMR analyses of recombinant NUPR1 showed absence of a stable secondary structure. NUPR1 binds DNA weakly as shown by electrophoretic mobility shift assay, without apparent preference for DNA sequences. It has been shown that human NUPR1 is a substrate for protein kinase A, and phosphorylated NUPR1 has a higher content of secondary structure and binding to DNA [4]. Based on these observations, NUPR1 plays an architectural role in transcription. Previous studies have shown that modulating NUPR1 expression affects tumor cell proliferation, cycle or apoptosis, such as liver cancer [6], pancreatic cancer [7], breast cancer and glioma [8]. NUPR1 also binds Jab1 to form complex as a component of COP9, which is a key factor modulating translocation p27 CDK inhibitor from the nucleus to the cytoplasm [9]. But the mechanism of NUPR1 in regulating glioma cell cycle remains poorly understood.

In this study, the ability to affect glioma cell cycle was explored by knocking down the expression of NUPR1 in U87 and U251 cells in vitro and in vivo. The role of NUPR1 in the development of human gliomas remains to be fully understood, which may provide new targeted therapies.

\section{Patients and methods}

Cell culture and reagents. The human glioma U87 and U251 cell lines were supplied by the Cell Bank of Shanghai Institute of Cell Biology, Chinese Academy of the Sciences. 
The cells were cultured in Dulbecco's modified Eagle medium (DMEM, Corning, USA) supplemented with $10 \%$ fetal bovine serum (FBS, Ausbian, Australia) at $37^{\circ} \mathrm{C}$ in $5 \%$ $\mathrm{CO}_{2}$. Used antibodies: rabbit anti-human NUPR1 antibody (Cat: sc-30184, Santa Cruz, USA), anti-rabbit IgG (Cat: 7074P2, Santa Cruz, USA) secondary antibodies as well as cell cycle regulation protein antibodies p27Kip1 (Cat: 3686p, Cell Signaling Technology, USA), CDK2 (Cat: 2561, Cell Signaling Technology, USA) and cyclin E (Cat: 4136, Cell Signaling Technology, USA) and anti-GAPDH (Cat: 600041-lg, Proteintech, USA).

Patients and tissue samples. All of the paraffinembedded samples, including glioma tissues (acquired during glioma excision operations), were obtained from the Department of Neurosurgery of the First Affiliated Hospital of Dalian Medical University, Liaoning, China. This study was approved by the Human Ethics Committee of the First Affiliated Hospital of Dalian Medical University (approval number: LCKY2014-20). All procedures performed in this study involving human participants were in accordance with the ethical standards of the institutional research committee and the 1964 Helsinki Declaration and its later amendments; animal ethics in accordance with the EU Directive 2010/63/ EU for animal experiments and the National Institutes of Health guide for the care and use of laboratory animals for the care and use of laboratory animals. This study conformed to the institutional medical requirements and written informed consent was obtained from all of the enrolled patients. After resection, the frozen specimens of tumor in nude mice were stored at $-80^{\circ} \mathrm{C}$ until RNA extraction.

Cells infection. Control RNA sequence (TTCTCCGAACGTGTCACGT) and shRNAs targeting NUPR1 (NUPR1-shRNA1: 5'-CTGGTGACCAAGCTGCAGA-3'; NUPR1-shRNA2: 5'-CATGCCTATGCCCACTTCA-3') were designed by GeneChem (shCtrl: No. LVPSC3741, shNUPR1: No. LVpGCSIL-004PSC1501-1, GeneChem, China). According to the infection efficiency, NUPR1shRNA1 was chosen for subsequent experiments. U251 and U87 cell suspensions of $5 \times 10^{4}$ cells were cultured in six-well plates for one day and then infected with lentiviruses at a set multiplicity of infection (MOI). The medium was changed after $8 \mathrm{~h}$, and the cells were screened using puromycin (2 $\mu \mathrm{g} / \mathrm{ml}$ ) if there were more than $80 \%$ GFP-fluorescent cells observed under a fluorescent microscope (OLYMPUS IX71) after infection for three days. Knockdown efficiency of U87 and U251 cells infected with a lentiviral vector was detected by quantitative real-time PCR and western blot.

Quantitative real-time PCR. We used the $2^{-\Delta \Delta C T}$ method to analyze the relative expression levels. The primer sequences of NUPR 1 were 5'-AGCCTGGATGAATCTGACCTCTAT-3' (forward) and 5'-GGTGTTGGCAGCAGCTTCTC-3' (reverse). The primer sequences of GAPDH, used as an internal control, were 5'-GCACCGTCAAGGCTGAGAAC$3 \mathrm{v}$ (forward) and 5'-TGGTGAAGACGCCAGTGGA-3' (reverse).
Western blot. Protein quantification was measured by the BCA method (Cat: PC0020, Solarbio, China). Equal amounts of protein were separated by SDS-PAGE (15\%), transferred to PVDF $(0.2 \mu \mathrm{m}$, Millipore, USA), and then blocked with 5\% non-fat milk. Membranes were incubated with rabbit antihuman NUPR1 antibody overnight at $4{ }^{\circ} \mathrm{C}$ and incubated in secondary antibodies for $2 \mathrm{~h}$ after washing with TBS the next day. Protein bands were detected by enhanced chemiluminescence (ECL) with Bio-Spectrum Gel Imaging System (UVP, USA).

Immunohistochemistry (IHC). Paraffinized sections were dipped in xylene for $15 \mathrm{~min}$ and then rehydrated in a graded ethanol series for $5 \mathrm{~min}$ per solution. Antigen retrieval was performed by heating the sections in a citrate-buffered solution $(0.01 \mathrm{M}, \mathrm{pH} 6.0)$ in a microwave oven at thawing temperature for $20 \mathrm{~min}$. Endogenous peroxidase activity was eliminated via incubation in 3\% hydrogen peroxide diluted in deionized water for $15 \mathrm{~min}$. After blocking with goat serum (ZSGB-BIO), the sections were incubated with a rabbit anti-human P27, CDK2, and cyclin E antibody (1:100) overnight at $4{ }^{\circ} \mathrm{C}$. The primary antibody was detected with a biotin-labeled goat anti-rabbit antibody (ZSGB-BIO) for $20 \mathrm{~min}$ at room temperature after washing the slides with phosphate-buffered saline (PBS, pH 7.4). Subsequently, the sections were incubated in horseradish peroxidase (HRP) for $15 \mathrm{~min}$. Finally, chromogenic reactions were performed using 3,3'-diaminobenzidine (DAB, ZSGB-BIO) and hematoxylin, and then, the samples were analyzed using a biomicroscope (LEICA, DM6000-B, Germany).

Animals and tumorigenesis assay. The procedures and animal care were in accordance with NIH Guidelines approved by the Ethics Committee of The First Affiliated Hospital of Dalian Medical University in China. About five weeks old female nude mice (BALB/c-nu/nu) were bought and raised at the Center of Experimental Animals, Dalian Medical University (China). The tumors were removed from the oxter of nude mice of the xenograft model established by subcutaneous injection of shNUPR1 U87 or shPLV-Ctrl U87 cells (about $1 \times 10^{7}$ cells) to the back after one month. Quantitative real-time PCR and western blot were used to detect NUPR1 expression levels between shNUPR1 and shPLV-Ctrl tumors.

Analysis of the cell cycle via FACS. Infected cells seeded in the 6-well plates were digested, resuspended and then centrifuged at $1300 \mathrm{rpm}$ for $5 \mathrm{~min}$ when the confluence reached $80 \%$. After washing the cells with D-Hanks buffer ( $\mathrm{pH} \sim 7.2-7.4)$, the cells were fixed with cold $70 \%$ ethanol for approximately $1 \mathrm{~h}$, centrifuged at $1300 \mathrm{rpm}$ for $5 \mathrm{~min}$ and washed with D-Hanks buffer. Finally, the cells were stained with $1 \mathrm{ml}$ of propidium iodide (PI, Sigma, Cat: P4170) and analyzed using a FACScan flow cytometer (Guava easyCyte HT, Millipore/Becton Dickinson, USA).

Statistical analysis. The quantified data are expressed as the means $\pm \mathrm{SD}$ of experiments performed in triplicate. GraphPad Prism 5.0 and Excel were used for statistical 
analysis and for graph drawing. Student's two-tailed t-test or one-way ANOVA was used to evaluate the differences between groups, and the Chi-squared test was used to analyze the differences between classified variables. A p-value $<0.05$ was considered statistically significant.

\section{Results}

Stable knockdown of NUPR1 in U87 and U251 cells by lentiviral infection. U87 and U251 cells, both derived from glioma, were selected to investigate the biological function of NUPR1 in regulating the glioma cell cycle. Contrasting with bright cells, GFP fluorescence was observed in more than $80 \%$ of the cells (Figure 1A). Real-time PCR and western blotting were used to observe the transfection efficiency of U87 and U251 cells infected with a lentiviral vector (shCtrl or shNUPR1). Compared to the shCtrl-transfected cells, the NUPR1-shRNA1 and NUPR1-shRNA2 transfected cells showed significantly reduced mRNA levels of NUPR1 $(\mathrm{p}<0.001)$, and NUPR1-shRNA1 indicated high transfection efficiency (Figure 1B), also confirmed by western blotting (Figure 1C).

Knockdown of NUPR1 arrested the cell cycle in the G0/G1 phase in U87 and U251 cells via P27. The effect of NUPR1 knockdown on the cell cycle distribution in U87 and U251 cells was explored by fluorescence-activated cell sorting plus propidium iodide (FACS-PI) staining. As shown in Figure 2A, compared with the shCtrl group, the shNUPR1 group exhibited a greater percentage of U87 cells in the G0/ G1 phase (average $46.75 \%$ vs. $36.63 \%$, p $<0.001$ ) and a lower percentage of cells in the S and G2 phases (average $38.13 \%$ vs. $41.79 \%$ and $15.12 \%$ vs. $21.58 \%$, respectively, $\mathrm{p}<0.05$ ), suggesting that the NUPR1-silenced U87 cells were arrested in the G0/G1 phase. In U251cells, the results revealed $48.36 \%$ of G0/G1 phase cells in the shNUPR 1 cells and $39.74 \%$ in the PLV-Ctr cells $(\mathrm{p}<0.01)$, and a lower percentage of cells in the $\mathrm{S}$ and G2 phases in the shNUPR1 cells (average $36.65 \%$ vs. $40.59 \%$ and $15.41 \%$ vs. $19.67 \%$, respectively, $\mathrm{p}<0.05$, Figure $2 \mathrm{~B})$. This result revealed that the NUPR1-silenced U87 and U251 cells were significantly arrested in the G0/G1 phase. The expression levels of cell cycle regulation proteins p27Kip1, CDK2, and cyclin $\mathrm{E}$ were determined using western blot analysis (Figure 2C). Our results suggested that low NUPR1 expression could reduce CDK2 and cyclin E expression while enhancing the expression of p27Kip1.

The expression levels demonstrate a negative correlation between P27 and NUPR1 in glioma tissues. To measure the expression of p27Kip, CDK2, and cyclin E in glioma tissues, IHC staining was performed (Figure 3 ). In this study, we found that p27Kip1 was lowly expressed in high-grade glioma tissues with positive NUPR1 expression and highly expressed in low-grade glioma tissues with negative NUPR1 expression (Figure 3A), however, the expression level of CDK2 and cyclin E were increased in high-grade glioma tissues with positive NUPR1 expression and decreased in low-grade glioma tissues with negative NUPR1 expression (Figures 3B, 3C).

Establishment of nude mouse xenograft. In our previous study, it was shown that knockdown of NUPR1 suppresses cell tumorigenicity in vivo. The tumors were removed from nude mice after the establishment of nude mouse xenograft for 30 days (Figure 4A). In this study, quantitative real-time PCR and western blot were performed to detect NUPR1 mRNA and protein expression levels in shNUPR1 and
A
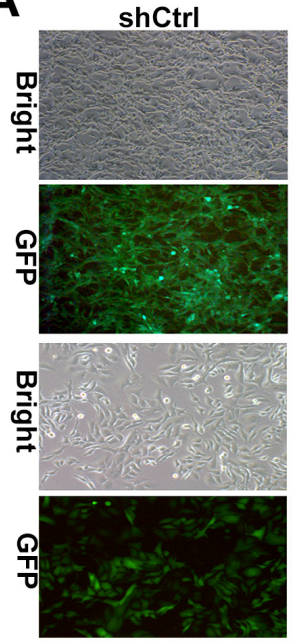

shNUPR1
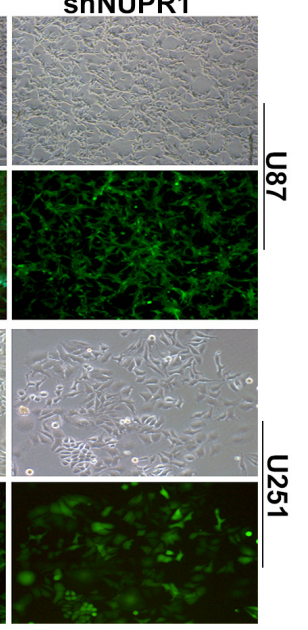

B

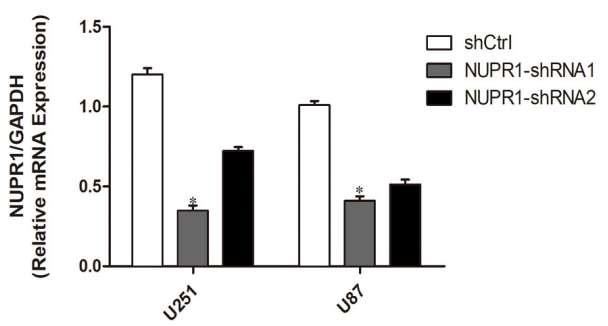

C

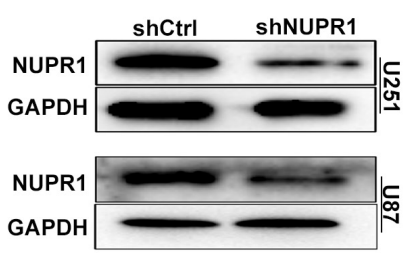

Figure 1. Lentivirus-mediated gene silencing of NUPR1 in the U87 and U251 human glioma cells. A) Infection efficiency in the U87 and U251 cells is shown by representative images of bright and GFP fluorescence after 3 days of lentiviral infection (magnification: 100 $\times$ ). B) The $\mathrm{qRT}$-PCR assay was used to detect the transcriptional levels of the NUPR1 gene after U87 and U251 cells were stably infected with shCtrl or NUPR1-shRNA. C) Expression analyses of NUPR1 protein in shCtrl group and NUPR1-shRNA group are shown by western blot assays. GAPDH was used as a loading control. The data are presented as the means \pm SD for the three independent experiments $(\mathrm{p}<0.05)$. 
A
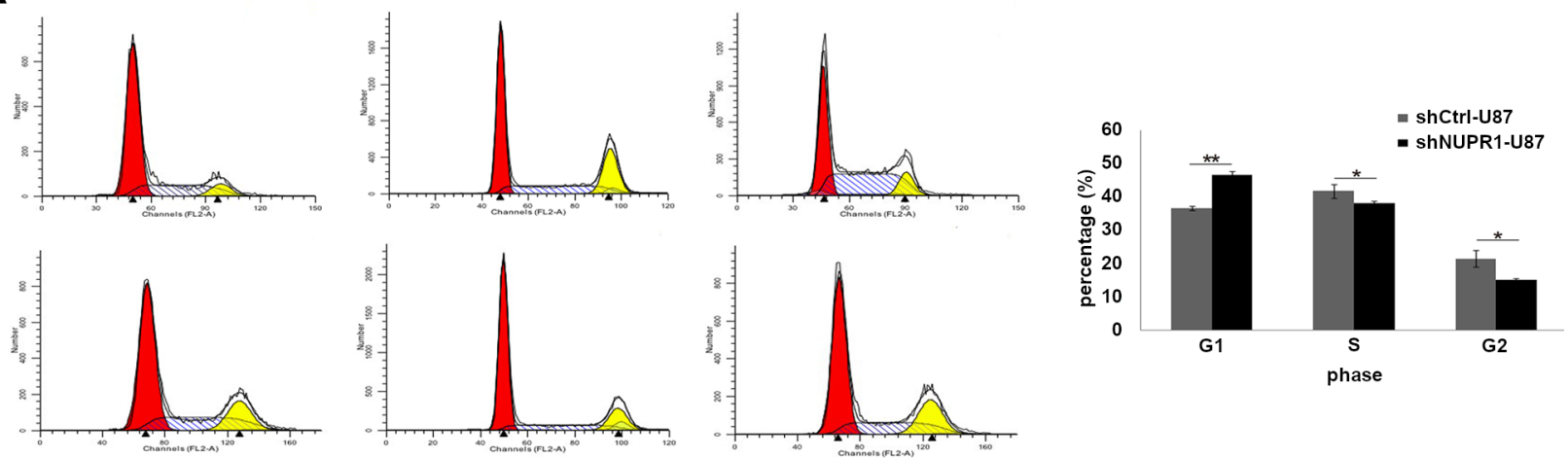

B
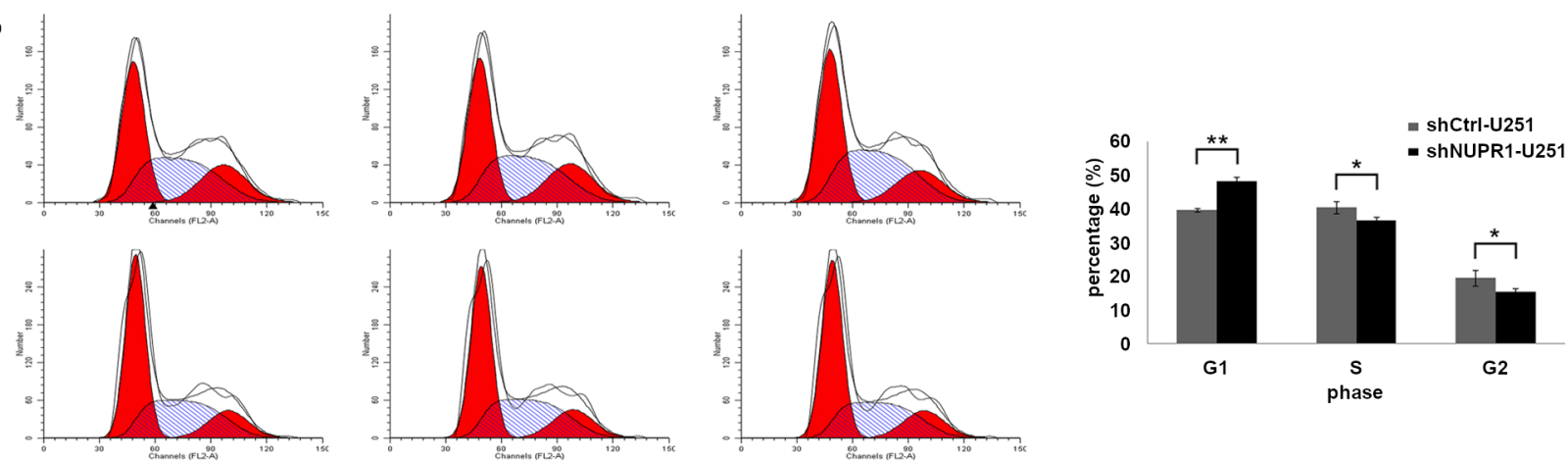

C

U87

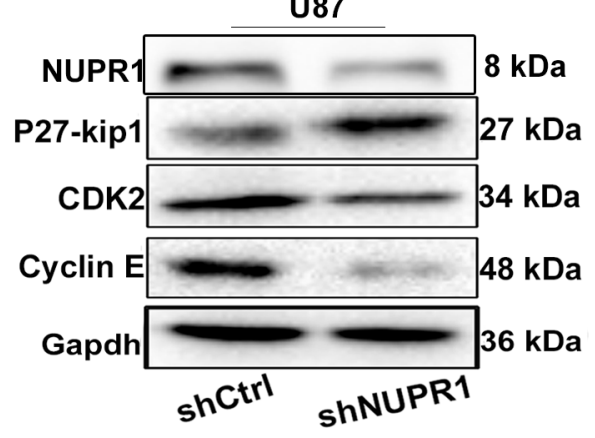

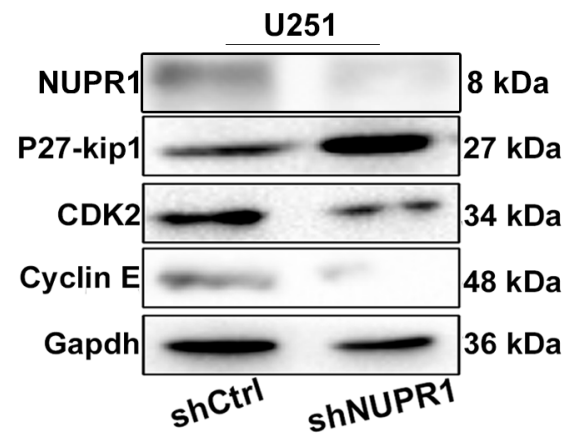

Figure 2. Knockdown of NUPR1 blocks cell cycle progression in glioma cells. The cell cycle distribution was performed via fluorescence-activated cell sorting (FACS). A) The proportion of U87 cells in the G0/G1 phase was increased in the shNUPR1 group compared to the shCtrl group (p<0.001). B) Compared with the shCtrl group, the population of U251 cells in the G0/G1 in the shNUPR1 group (p<0.001). C) Knockdown of NUPR1 reduced the expression of CDK2 and cyclin E, however, P27 was highly expressed. GAPDH was used as a loading control. Data are presented for three independent experiments.

shPLV-Ctrl tumors. The results of PCR implied that the expression of NUPR1 mRNA in the shNUPR1-xenografted tumors was reduced compared with the shCtrl-xenografted tumors (Figure 4B), and western blotting indicated the same result (Figure 4C).

Knockdown of NUPR1 arrested the cell cycle in the G0/ G1 phase via P27 in vivo. In the xenografted tumors, the results of IHC staining showed that the expression of p27Kip1 was low in the shNUPR1-xenografted tumors compared with the shCtrl-xenografted tumors (Figure 5A), however, CDK2 and cyclin E showed opposite results (Figures 5B, 5C), which correspond to the same result as glioma tissues and U87/ U251 cells.

\section{Discussion}

Previous studies revealed that the expression levels of NUPR1 are related to tumor progress and prognosis in a variety of tumors [10]. In the nervous system, NUPR1 is a key gene involved in sensitizing cultured serum-deprived astro- 

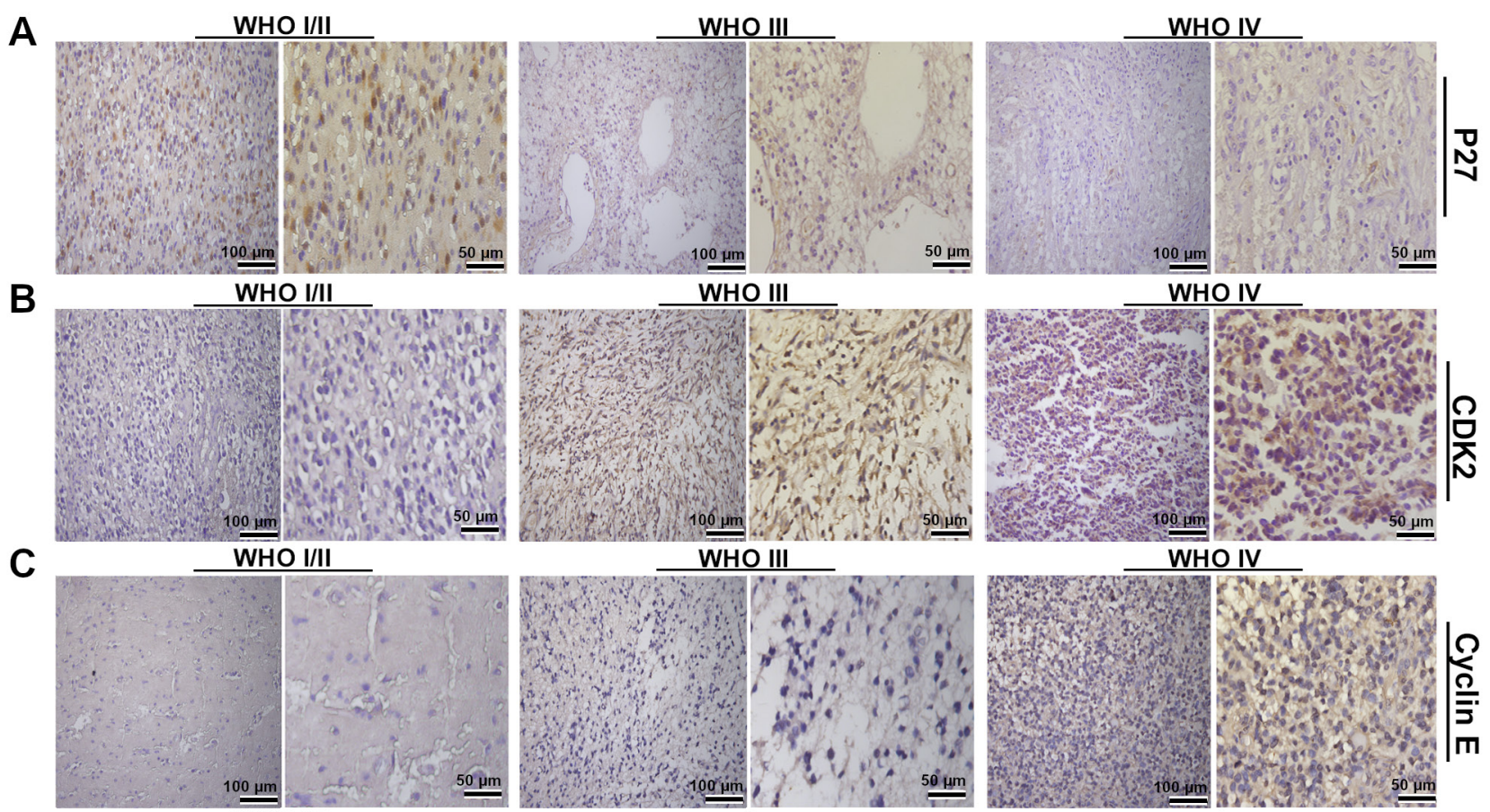

D
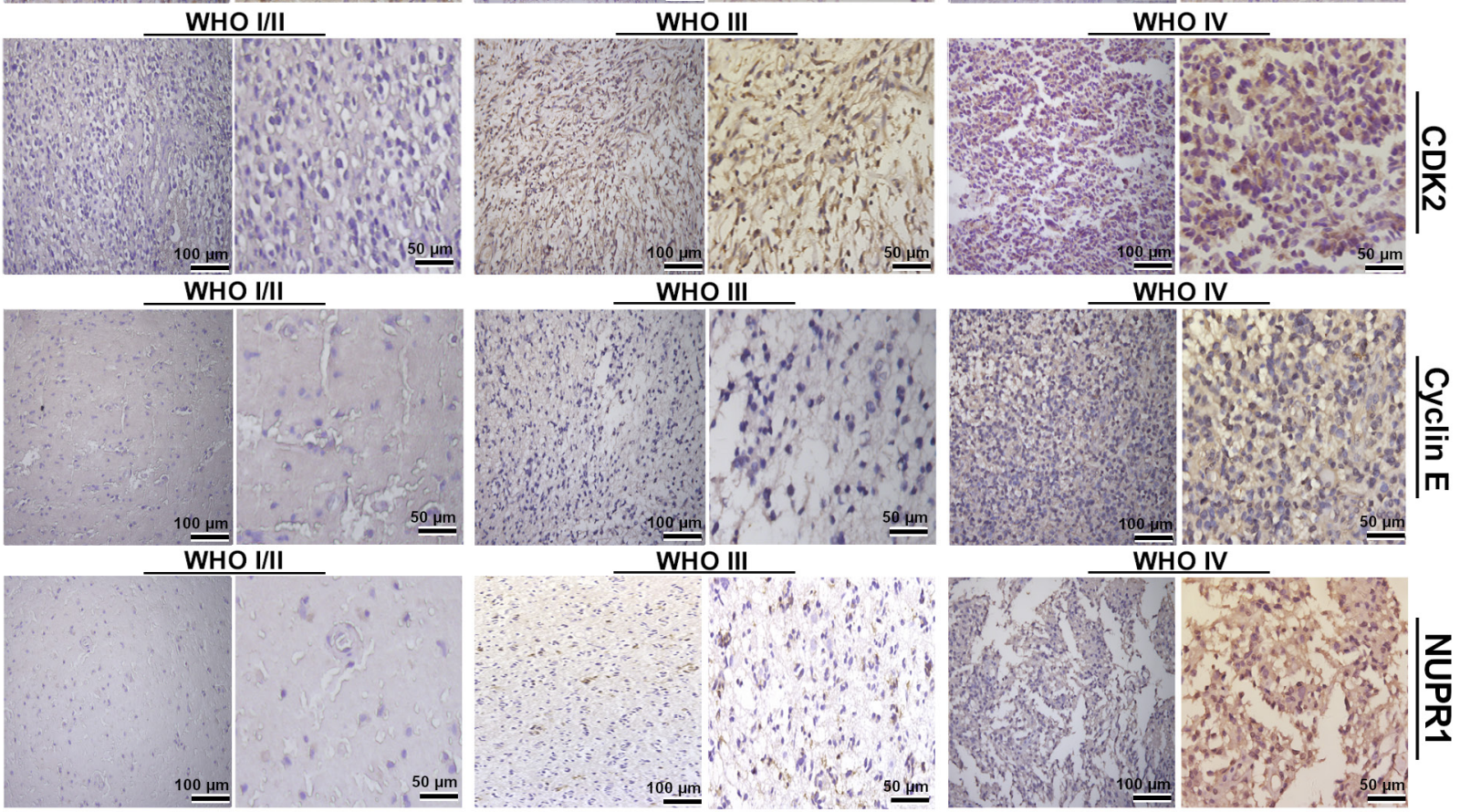

Figure 3. Representative glioma cases with high NUPR1 expression showing immunohistochemical (IHC) staining for P27, CDK2, cyclin E, and NUPR1 according to the tumor WHO histological grade. A) P27 expression levels in WHOI/II glioma tissues with negative NUPR1 expression, and P27 expression levels in WHO III and WHO IV grade glioma tissues with positive NUPR1 expression. B, C) CDK2 and cyclin E expression levels are different in WHO I/II glioma tissues with negative NUPR1 expression, CDK2 and cyclin E expression levels are different in WHO III and WHO IV glioma tissues with positive NUPR1 expression. D) NUPR1 expression levels in glioma tissues. (original magnification: 100×, 50×)

A
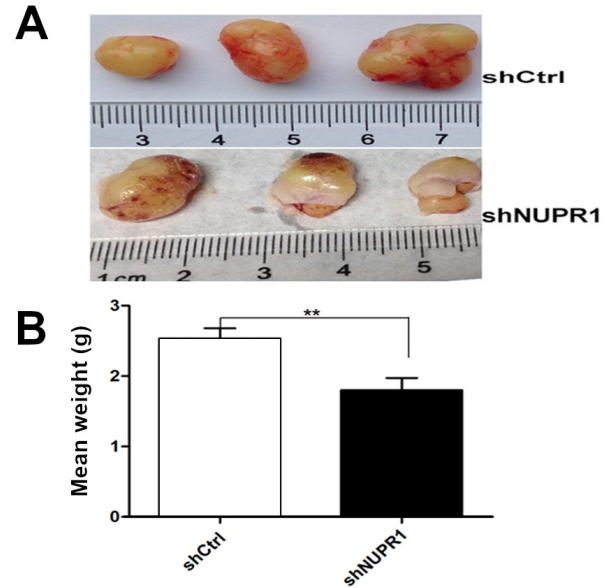
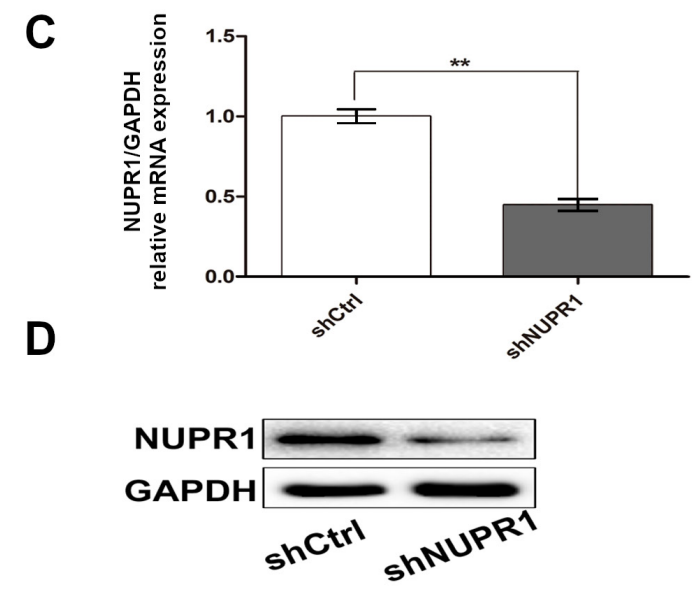

Figure 4. Cell tumourigenicity via subcutaneously injecting PLV-Ctrl-U87 and sh-NUPR1-U87 cells in female nude mice. A) The tumors were removed from mice injected with shPLV-Ctrl and shNUPR1 U87 cells after one month. B) The tumorigenicity of shNUPR1 cells was reduced in vivo compared with the shCtrl group. C) The qRT-PCR assay was used to detect NUPR1 mRNA levels in subcutaneous tumors of mice injected with PLV-Ctrl cells and shNUPR1. D) Western blot assay was performed to detect NUPR1 protein in the shCtrl group and the NUPR1-shRNA group. GAPDH was used as a loading control. Six mice were used for each treatment. Data are presented for three independent experiments $\left({ }^{\star *} \mathrm{p}<0.01\right)$. 
cytes to oxidative stress [11]. Recent data from astrocytes demonstrated that NUPR1 suppression led to the induction of heme oxygenase 1 (HO-1) [12]. In gliomas, NUPR1 was required for the efficient induction of the endoplasmic reticulum stress-related genes activating transcription factor 4 (ATF4), C-homologous protein (CHOP), and Tribbles Homologue3 (TRB3) [13], and NUPR1 could modulate the apoptosis of glioma C6 cells with TRB3 in a cannabinoid-dependent manner via the ERK pathway [14]. Taken together, these observations suggested that NUPR1 expression under non-stress conditions might be protective for the cell, whereas its abnormal expression might result in a worse fate for the cell. In our previous experiment about NUPR1 in glioma, it was shown that NUPR1 plays an important role in the growth and migration of glioma cells [15], however, the specific mechanism is poorly understood. In the present study, FACS analysis demonstrated that LV-shNUPR1infected U87 cells were arrested in the G0/G1 phase (46.75\%) however, only $36.63 \%$ of the control cells were in the G0/G1 phase, and the infected U251 cells also showed the same result $(48.36 \%$ vs. $39.74 \%)$. These results revealed that low expression levels of NUPR1 significantly arrested the cell cycle in the G0/G1 phase.
In HepG2 cells, knockdown of NUPR1 expression arrested HepG2 cells in the G1 phase [16]. A cell re-entering the cell cycle or undergoing cell cycle arrest is depended on the stress-associated pathways in the genetic response. As a stress factor, NUPR1 regulates the cell cycle in fibroblasts [17]. Comparing to NUPR1-deficient fibroblasts, normal fibroblasts express more cell cycle arrests via serum deprivation. Moreover, it showed that cyclin-dependent kinase inhibitor p27 is decreased in NUPR1-deficient fibroblasts. Indeed, researchers found the mechanism by which NUPR1 regulates the degradation of p27. NUPR1 binds Jab1 to form complex as a component of the COP9 which is a key factor modulating translocation p27 CDK inhibitor from the nucleus to the cytoplasm where it is degraded $[9,18]$. As we know, p27Kip1 is a critical component of the cell cycle machinery as an inhibitor of Cdk2-cyclin E, and it controls cell S-phase entry and G1-phase exit during tumorigenesis $[19,20]$. Therefore, it is suspected that NUPR 1 could affect the biological functions of tumor cells by regulating cell circle in glioma via p27.

In the present study, results of western blot showed that the expression level of p27 was increased in U87 and U251 cells infected by shNUPR1, while CDK2 and cyclin E were
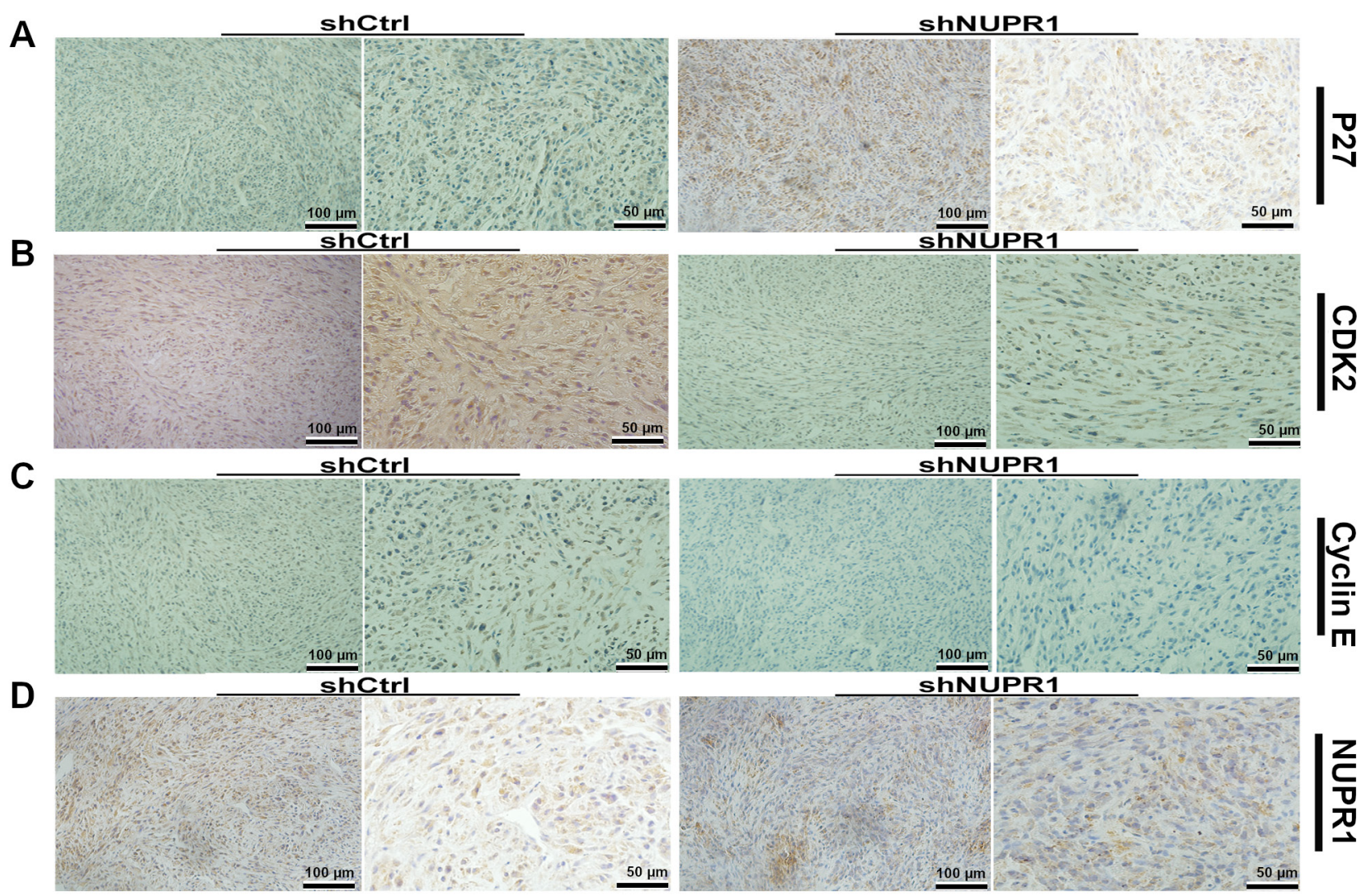

Figure 5. IHC staining for P27, CDK2, and cyclin E in subcutaneous tumors of mice. A) IHC staining showed the P27 expression in shCtrl and shNUPR1 subcutaneous tumors. B) IHC staining showed the CDK2 expression in shCtrl and shNUPR1 subcutaneous tumors. C) IHC staining showed the cyclin E expression in shCtrl and shNUPR1 subcutaneous tumors. D) IHC staining showed the NUPR1 expression in shCtrl and shNUPR1 subcutaneous tumors. (original magnification: $100 \times, 50 \times$ ) 
decreased in vitro. Also, the expressions of p27Kip1, CDK2, and cyclin $\mathrm{E}$ in different WHO grade gliomas were detected using IHC, which revealed that p27Kip1 was highly expressed in low-grade glioma tissues with negative NUPR1 expression and CDK2 and cyclin E were lowly expressed while in high-grade glioma tissues with positive NUPR1 expression, p27Kip1 was lowly expressed. In the xenografted tumors, IHC staining showed that the expression of p27Kip1 was low in the shNUPR1-xenografted tumors compared with the shCtrl-xenografted tumors. This is in accordance with the result of knockdown of NUPR1 that induces cell cycle arrest in the G0/G1 phase in U87 and U251 cells via increasing P27 expression in vitro. But the result in our research is different from that p27 is expressed at a low level in NUPR1-deficient cells in fibroblasts. The discrepancy may be a result of several reasons. First, previous studies have shown that P27 protein is mainly located in the nucleus [20], in gliomas, positive nuclei expression of p27 decreased in number and staining intensity with the increasing degree of histological malignancy in gliomas [21], and increased in cytoplasm of highgrade glioma. Second, NUPR1 binds Jab1 to form complex as a component of the COP9, which is a key factor modulating translocation p27 from the nucleus to the cytoplasm where it is degraded [9], thus NUPR1-deficiency could suppress translocation p27 from the nucleus and p27 expression level is increased in NUPR1-deficient glioma cells. In conclusion, knockdown of NUPR1 induces cell cycle arrest in the G0/G1 phase in glioma cells via P27 in vivo and in vitro.

Acknowledgments: This study is sponsored by Dalian medical science research program (No. 1912025) and Liaoning science and technology plan project (2019-ZD-0646).

\section{References}

[1] WIRSCHING HG, WELLER M. The Role of Molecular Diagnostics in the Management of Patients with Gliomas. Curr Treat Options Oncol 2016; 17: 51. https://doi.org/10.1007/ s11864-016-0430-4

[2] JIANG T, MAO Y, MA W, MAO Q, YOU Y et al. CGCG clinical practice guidelines for the management of adult diffuse gliomas. Cancer Lett 2016; 375: 263-273. https://doi. org/10.1016/j.canlet.2016.01.024

[3] VASSEUR S, MALLO GV, GARCIA-MONTERO A, ORTIZ EM, FIEDLER F et al. Structural and functional characterization of the mouse $\mathrm{p} 8$ gene: promotion of transcription by the CAAT-enhancer binding protein alpha (C/EBPalpha) and $\mathrm{C} / \mathrm{EBPb}$ ta trans-acting factors involves a C/EBP cisacting element and other regions of the promoter. Biochem J 1999; 343: 377-383.

[4] ENCINAR JA, MALLO GV, MIZYRYCKI C, GIONO L, GONZALEZ-ROS JM et al. Human p8 is a HMG-I/Y-like protein with DNA binding activity enhanced by phosphorylation. J Biol Chem 2001; 276: 2742-2751. https://doi. org/10.1074/jbc.M008594200
[5] CANO CE, SANDI MJ, HAMIDI T, CALVO EL, TURRINI $\mathrm{O}$ et al. Homotypic cell cannibalism, a cell-death process regulated by the nuclear protein 1, opposes to metastasis in pancreatic cancer. EMBO Mol Med 2012; 4: 964-979. https:// doi.org/10.1002/emmm.201201255

[6] LEE YK, JEE BA, KWON SM, YOON YS, XU WG et al. Identification of a mitochondrial defect gene signature reveals NUPR1 as a key regulator of liver cancer progression. Hepatology 2015; 62: 1174-1189. https://doi.org/10.1002/ hep. 27976

[7] CANO CE, HAMIDI T, GARCIA MN, GRASSO D, LONCLE C et al. Genetic inactivation of Nuprl acts as a dominant suppressor event in a two-hit model of pancreatic carcinogenesis. Gut 2014; 63: 984-995. https://doi.org/10.1136/ gutjnl-2013-305221

[8] LI J, REN S, LIU Y, LIAN Z, DONG B et al. Knockdown of NUPR1 inhibits the proliferation of glioblastoma cells via ERK1/2, p38 MAPK and caspase-3. J Neurooncol 2017; 132: 15-26. https://doi.org/10.1007/s11060-016-2337-0

[9] MALICET C, HOFFMEISTER A, MORENO S, CLOSA D, DAGORN JC et al. Interaction of the stress protein $\mathrm{p} 8$ with Jab1 is required for Jab1-dependent p27 nuclear-to-cytoplasm translocation. Biochem Biophys Res Commun 2006; 339: 284-289. https://doi.org/10.1016/j.bbrc.2005.11.018

[10] LEE YK, WOO HG, YOON G. Mitochondrial defectresponsive gene signature in liver-cancer progression. BMB Rep 2015; 48: 597-598. https://doi.org/10.5483/bmbrep.2015.48.11.180

[11] CARRACEDO A, EGIA A, GUZMAN M, VELASCO G. p8 Upregulation sensitizes astrocytes to oxidative stress. FEBS Lett 2006; 580: 1571-1575. https://doi.org/10.1016/j.febslet.2006.01.084

[12] WEIS S, BIELOW T, SOMMERER I, IOVANNA J, MALICET C et al. P8 deficiency increases cellular ROS and induces HO-1. Arch Biochem Biophys 2015; 565: 89-94. https://doi. org/10.1016/j.abb.2014.11.007

[13] CARRACEDO A, LORENTE M, EGIA A, BLAZQUEZ C, GARCIA $S$ et al. The stress-regulated protein p8 mediates cannabinoid-induced apoptosis of tumor cells. Cancer Cell 2006; 9: 301-312. https://doi.org/10.1016/j.ccr.2006.03.005

[14] LORENTE M, CARRACEDO A, TORRES S, NATALI F, EGIA A et al. Amphiregulin is a factor for resistance of glioma cells to cannabinoid-induced apoptosis. Glia 2009; 57: 1374-1385. https://doi.org/10.1002/glia.20856

[15] BAK Y, SHIN HJ, BAK IS, YOON DY, YU DY. Hepatitis $B$ virus $X$ promotes hepatocellular carcinoma development via nuclear protein 1 pathway. Biochem Biophys Res Commun 2015; 466: 676-681. https://doi.org/10.1016/j. bbrc.2015.09.082

[16] VASSEUR S, HOFFMEISTER A, GARCIA-MONTERO A, MALLO GV, FEIL R et al. p8-deficient fibroblasts grow more rapidly and are more resistant to adriamycin-induced apoptosis. Oncogene 2002; 21: 1685-1694. https://doi. org/10.1038/sj.onc.1205222

[17] CANO CE, HAMIDI T, SANDI MJ, IOVANNA JL. Nupr1: the Swiss-knife of cancer. J Cell Physiol 2011; 226: 14391443. https://doi.org/10.1002/jcp.22324 
[18] WANG Y, WANG Y, XIANG J, JI F, DENG Y et al. Knockdown of CRM1 inhibits the nuclear export of p27Kip1 phosphorylated at serine 10 and plays a role in the pathogenesis of epithelial ovarian cancer. Cancer Lett 2014; 343: 6-13. https://doi.org/10.1016/j.canlet.2013.09.002

[19] HNIT SST, XIE C, YAO M, HOLST J, BENSOUSSAN A et al. p27Kip1 signaling: Transcriptional and post-translational regulation. Int J Biochem Cell Biol 2015; 68: 9-14. https:// doi.org/10.1016/j.biocel.2015.08.005
[20] SHI J, ZHANG L, SHEN A, ZHANG J, WANG Y et al. Clinical and biological significance of forkhead class box $\mathrm{O} 3 \mathrm{a}$ expression in glioma: mediation of glioma malignancy by transcriptional regulation of p27kip1. J Neurooncol 2010; 98: 57-69. https://doi.org/10.1007/s11060-009-0045-8

[21] HE SM, ZHAO ZW, WANG Y, ZHAO JP, WANG L et al. Potential role of Jun activation domain-binding protein 1 and phosphorylated p27 expression in prognosis of glioma. Brain Tumor Pathol 2012; 29: 3-9. https://doi.org/10.1007/s10014011-0061-1 